\title{
MULTIVARIATE REARRANGEMENTS AND BANACH FUNCTION SPACES WITH MIXED NORMS
}

\author{
BY
}

\author{
A. P. BLOZINSKI
}

\begin{abstract}
Multivariate nonincreasing rearrangement and averaging functions are defined for functions defined over product spaces. An investigation is made of Banach function spaces with mixed norms and using multivariate rearrangements. Particular emphasis is given to the $L(P, Q ; *)$ spaces. These are Banach function spaces which are in terms of mixed norms, multivariate rearrangements and the Lorentz $L(p, g)$ spaces. Embedding theorems are given for the various function spaces. Several well-known theorems are extended to the $L(P, Q ; *)$ spaces. Principal among these are the Strong Type (Riesz-Thorin) Interpolation Theorem and the Convolution (Young's inequality) Theorem.
\end{abstract}

\section{Introduction.}

1.1. Let $f(x), x=\left(x_{1}, \ldots, x_{n}\right)$ be a measurable function on a totally $\sigma$-finite product space $(\Omega, \mu)=\left(\times \Omega_{i}, \times \mu_{i}\right)$. Consider complex valued measurable functions $f(x)$ which are finite almost everywhere and which, for some $\sigma>0, \lambda_{\gamma}(\sigma)=$ $\mu\{x:|f(x)|>\sigma\}$ is finite. The nonincreasing rearrangement of $f(x)$ on $(0, \infty)$ is given by $f^{*}(u)=\inf \left\{\sigma: \lambda_{f}(\sigma) \leqslant u\right\}, u>0$. Since many operations with functions defined on product spaces are iterative, C. J. Neugebauer suggested that it should be possible to obtain multivariate rearrangements by such a process. We make our definition in \$2. As defined, the function $f^{*}(t), t=\left(t_{1}, \ldots, t_{n}\right), t_{i}>0, i=$ $1, \ldots, n$, will be a rearrangement of the function $f(x)$ which is nonincreasing in each of the variables $t_{i}$. The method of definition is iterative and consistent with the one variable method. The function $f^{*}(t)$ is shown to enjoy the same basic properties of its one dimensional counterpart $f^{*}(u), u>0$. The functions $f(x)$ and $f^{*}(t)$ are shown to be equimeasurable with respect to their product measures. This in turn leads to a basic inequality relating the functions $f^{*}(t), t=\left(t_{1}, \ldots, t_{n}\right)$, and $f^{*}(u)$, $u>0$. Importantly, a method is obtained for putting the terms of multivariate simple functions in decreasing order.

The mixed norm spaces $L^{p}$ were introduced by Benedek and Panzone [1]. Banach function spaces were introduced by Luxemburg [12] and Banach function spaces which are rearrangement invariant by Luxemburg [13]. In §3, we construct the Banach function spaces with mixed norm which are appropriate to multivariate rearrangements. Comparisons are made between these spaces and Banach function spaces with mixed norms in the ordinary sense (of [1]). The basic properties of the

Received by the editors October 22, 1979 and, in revised form, January 11, 1980.

1980 Mathematics Subject Classification. Primary 46E35; Secondary 46E35, 46A96, 26A33, 28 A25.

Key words and phrases. Multivariate rearrangements, averaging operators, Banach function spaces, Lorentz $L(p, q)$ spaces, mixed norm spaces. 
various mixed norm spaces are established. In a final result of the section, the various mixed norm spaces are placed in context as intermediate between the tensor projective cross product of their underlying function spaces and the space of bounded integral operators between Banach function spaces.

The Lorentz $L(p, q)$ spaces were introduced by G. G. Lorentz. In [11], where R. A. Hunt collects his interpolation theorems, the $L(p, q)$ spaces are investigated in some detail. In some respects the Lorentz $L(p, q)$ spaces play a central role in the study of Banach function spaces. Oftentimes, the methods used to investigate the $L(p, q)$ spaces are useful for obtaining results for more generalized Banach function spaces. And results for the $L(p, q)$ spaces of ten have natural analogues in the more generalized settings. The remainder of the paper is devoted to an investigation of multivariate versions of the $L(p, q)$ spaces. These are the Lorentz $L(P, Q)$ and $L(P, Q ; *)$ spaces. The former are mixed norm spaces in the ordinary sense (of [1], using $L(p, q)$ spaces). The latter are mixed norm spaces using multivariate rearrangements and the $L(p, q)$ spaces. In $\$ 4$ the results of $\S \S 2$ and 3 are applied to define and investigate these particular spaces. Containment relationships are provided for the $L(P, Q), L(p, q)$ and $L(P, Q ; *)$ spaces.

$\S 5$ contains the applications and main results. These consist of several wellknown theorems which are extended to the $L(P, Q ; *)$ spaces. In these regards and as a main result, the Strong Type (Riesz-Thorin) Interpolation Theorem [11] is extended to the $L(P, Q ; *)$ spaces. The proof is made possible by the use of rearrangable simple functions, which are introduced in the paper, and use of the corresponding $L(p, q)$ proof given in Hunt [11]. Included also are extensions of the Convolution Theorem [16] and the Theorem on Fractional Integration [16].

Banach function spaces with mixed norm have been of continuing interest to several authors. Though much has been written on the subject, the use of multivariate rearrangements in the study of such spaces and our unified treatment are apparently new. In these regards we should mention the works of C. Ballester de Pereyra [7] and D. L. Fernandez [8]. In [8] Fernandez introduces the $L(P, Q ; *)$ spaces using the Peetre $K$-functional and for a main result extends an $L(P, Q)$ Marcinkiewicz type interpolation theorem of de Pereyra. Their interpolation theorems are in terms of four endpoint conditions (for dimension $n=2$ ). The RieszThorin Theorem mentioned above is of the sort in [1] where only two endpoint conditions are used. In this respect the two sets of results are noninclusive of each other. Also, some basic results for the $L(P, Q)$ and $L(P, Q ; *)$ spaces are presented in [8]. However, due to certain assumptions that the author makes, the proofs there are not always complete.

Beyond use of the above mentioned references [1], [11]-[13], the presentation presupposes only a knowledge of basic measure theory [9].

\section{Preliminaries.}

2.1. Averaging operators. We begin by extending some standard definitions and known facts about rearrangements of functions of one variable to the multivariate case. For the sake of simplicity, this will be done first for the case of two variables. Let $\Omega_{1}$ and $\Omega_{2}$ be nonnegative, separable, $\sigma$-finite measure spaces with measures $\mu$ 
and $\nu$ respectively. The spaces $\Omega_{1}$ and $\Omega_{2}$ are assumed to be either nonatomic or purely atomic with each of the atoms having equal measure. The functions $f(x, y)$ are complex valued and defined on the product space $\left(\Omega_{1} \times \Omega_{2}, \mu \times \nu\right)$; see [9]. It will often be convenient to suppress mention of a particular underlying measure. This will particularly be true whenever a given measure $\mu$ (or $\nu$ ) is replaced by Lebesgue measure $d m$ on $(0, \infty)$. When this occurs it will be clear from the context. With this in mind we will often put $\mu(E)=|E|$ and $d \mu=d x$ (similarly for the measures $\nu, d m$ and the product measures). If $|E| \geqslant s, s>0$, and the set $E$ depends on the parameter $s$, we put $E=E_{s}$. If in addition a second parameter is involved in the indexing of $E$, say $t, E$ will be written $E=E(s ; t)$.

The distribution function of $f(x, y)$ in the first variable and on $(0, \infty)$ is defined by

$$
\lambda_{f}(\sigma, y)=\mu\left\{x \in \Omega_{1}:|f(x, y)|>\sigma\right\}, \quad \sigma>0 .
$$

The nonnegative nonincreasing rearrangement of $f(x, y)$ in the first variable is defined by

$$
f^{*}(s, y)=\inf \left\{\sigma>0: \lambda_{f}(\sigma, y) \leqslant s\right\}, \quad s>0 .
$$

The averaging function of $f(x, y)$ in the first variable is defined by

$$
\bar{f}_{r}(s, y)=\sup _{E}\left[\frac{1}{|E|} \int_{E}|f(x, y)|^{r} d x\right]^{1 / r}
$$

where $E=E(s: y), E \subset \Omega_{1},|E| \geqslant s$. For future reference, note that the sets $E$ depend both on $s, s>0$, and the alternate variable $y$.

Some important properties of these functions are contained in our first lemma.

LEMMA 2.1. Let $E \subset \Omega_{1}$ be a measurable set; then

I. $\int_{E}|f(x, y)| d x \leqslant \int_{0}^{|E|} f^{*}(s, y) d s$, with equality if $E=\Omega_{1}$.

II. $\int_{E}|f(x, y) g(x, y)| d x \leqslant \int_{0}^{|E|} f^{*}(s, y) g^{*}(s, y) d s$.

III. $f^{*}(s, y)=\left[f^{*}(\cdot, y)\right]^{*}(s, y)$ and $\lambda_{f}(\sigma, y)=\lambda_{f^{*}(\cdot, y)}(\sigma, y)$. a.e.

IV. Put $\lambda_{f}=\lambda_{f}(\sigma, y)$ and $\lambda_{f^{*}}=\lambda_{f^{*}(\cdot, y)}(\sigma, y)$; then $\lambda_{\lambda_{f}}(s, y)=\lambda_{\lambda_{f}}(s, y)=f^{*}(s, y)$,

After obtaining a rearrangement of $f(x, y)$ with respect to the first variable, the new function $f^{*}(s, y)$, by holding $s$ fixed, can then be rearranged with respect to the second variable $y$. Doing this we obtain the function

$$
f^{*}(s, t)=\left[f^{*}(s, \cdot)\right]^{*}(t), \quad s>0, t>0,
$$

which is the nonnegative nonincreasing rearrangement of $f(x, y)$ in the first variable followed by its rearrangement with respect to the second variable. Throughout $0<s, t, u, v<\infty, x \in \Omega_{1} ; y \in \Omega_{2}$. Use of these variables with functions will be as with the functions $\left(f(x, y), f^{*}(s, y), f^{*}(s, t)\right)$ given above.

The order in which the rearrangement is taken is fundamental, because in general a different function is obtained if the order of rearrangement is reversed. This can be seen by considering the example $f(x, y)=\Sigma_{i, j}^{2,3} C(i, j) \chi_{E(i, j)}(x, y)$, where $E(i, j)=[i-1, i) \times[j-1, j)$ and $C(1,1)=1, C(1,2)=4, C(1,3)=3, C(2,1)=$ $5, C(2,2)=2, C(2,3)=6$. Here, the easiest way to obtain the rearrangement 
functions for $f(x, y)$ is to form the matrix array $(C(i, j))$. Rearrangement with respect to a given variable then corresponds to putting the entries of a given column or row in descending order.

Associated with a function $f(x, y)$ are two basic multivariate averaging operators

$$
\bar{f}_{r}(s, t)=\left[\overline{\bar{f}_{r}(s, \cdot)}\right]_{r}(t) \text { and } f_{r}^{* *}(s, t)=\left\{\frac{1}{s t} \int_{0}^{t} \int_{0}^{s} f^{* r}(u, v) d u d v\right\}^{1 / r} \text {, }
$$

where $\bar{f}_{1}(s, t)=\bar{f}(s, t)$ and $f_{1}^{* *}(s, t)=f^{* *}(s, t) ; s>0, t>0$.

The basic properties of the rearrangement function and the various averaging operators are contained in the next

LEMMA 2.2. I. $f^{*}, \bar{f}_{r}, f_{r}^{* *}$ are nonincreasing functions in $s$ and $t, s>0, t>0$.

II. If $|f(x, y)| \leqslant|g(x, y)|$, then $f^{*} \leqslant g^{*}, \bar{f}_{r} \leqslant \bar{g}_{r}$ and $f_{r}^{* *} \leqslant g_{r}^{* *}$.

III. $f^{*} \leqslant \bar{f}_{r} \leqslant f_{r}^{* *}, \bar{f}_{r_{1}} \leqslant \bar{f}_{r_{2}}$ and $f_{r_{1}}^{* *} \leqslant f_{r_{2}}^{* *}, 0<r_{1}<r_{2}<\infty, \overline{(f+g)} \bar{f}_{r} \leqslant \bar{f}_{r}+\bar{g}_{r}$, $1 \leqslant r<\infty$ and $\overline{(f+g)_{r}^{r}} \leqslant f_{r}^{r}+\bar{g}_{r}^{r}, 0<r<1$.

IV. $(f+g)^{*}\left(s_{1}+s_{2}, t_{1}+t_{2}\right) \leqslant f^{*}\left(s_{1}, t_{1}\right)+g^{*}\left(s_{2}, t_{2}\right)$.

Proof. Parts I and II follow directly from the one dimensional proofs and iteration. Part III also follows directly, except careful use is required of the notation and definitions. This is especially the case with respect to use of the underlying sets $E$ used in defining the averaging functions $\bar{f}_{r}$. For part IV, the one dimensional case implies $(f+g)^{*}\left(s_{1}+s_{2}, y\right) \leqslant f^{*}\left(s_{1}, y\right)+g^{*}\left(s_{2}, y\right)$. Therefore,

$$
(f+g)^{*}\left(s_{1}+s_{2}, t_{1}+t_{2}\right)<\left[f^{*}\left(s_{1}, \cdot\right)+g^{*}\left(s_{2}, \cdot\right)\right]^{*}\left(t_{1}+t_{2}\right) \text {. }
$$

After repeating the above steps, the proof follows.

We remark that in practice the functions $f^{*}$ and $f_{r}^{* *}$ are most convenient for defining norms (quasi-norms) on function spaces and working with norm type inequalities. In particular, the function $f_{r}^{* *}$ is especially suited for applications of the Hardy inequalities; see [4], [11]. The operator $\bar{f}_{r}$ is useful because it avoids difficulties which occur when the underlying measures are atomic and leads to a metric or a norm (a triangle inequality is satisfied) on the various function which will be considered. For some purposes $\bar{f}_{r}$ is more closely related to the function $f(x, y)$ than are the functions $f^{*}$ and $f_{r}^{* *}$. Moreover,

$$
t s \bar{f}(s, t) \simeq K(t ; K(s ; f(s, y))), \quad \text { where } K(u ; f)=\inf \left(\left\|f_{1}\right\|_{L^{1}}+u\left\|f_{2}\right\|_{L^{\infty}}\right)
$$

is the classical Peetre $K$-functional [4]. This shows that $\bar{f}_{r}(s, t)$ is useful for investigating and possibly obtaining multivariate versions of some of the known classical operators. For instance, see [4] or Lemma 5.2.

LEMMA 2.3. If $f(x, y)$ and $f_{k}(x, y), k=1,2, \ldots$, are positive functions such that $f_{k} \uparrow f$ pointwise, then $f_{k}^{*} \uparrow f^{*},\left(\bar{f}_{k}\right)_{r} \uparrow \bar{f}_{r}$ and $\left(f_{k}\right)_{r}^{* *} \uparrow f_{r}^{* *}$, pointwise a.e.

Proof. The monotonicity of the measure $\mu$ implies that, for $\sigma>0$, $\lambda_{f_{k}}(\sigma, y) \uparrow \lambda_{f}(\sigma, y)$ as $k$ tends to infinity. Repeating the procedure with Lebesgue measure on $[0, \infty)$ and still keeping $y$ fixed, $\lambda_{\lambda_{j_{k}}}(s, y) \uparrow \lambda_{\lambda_{j}}(s, y)$ as $k$ tends to infinity. By Lemma 2.1.IV, $f_{k}^{*}(s, y) \uparrow f^{*}(s, y)$ pointwise a.e. Finally, by holding $s$ fixed and 
repeating the procedure with respect to the second variable $y$, the conclusion follows for rearrangement functions.

The proof for the averaging functions follows by monotonicity and a straightforward application of Fatou's lemma. This completes the proof.

A function $f(x)$ defined over a measure space $(\Omega, \mu)$ is equimeasurable with a function $g(x)$ defined over a measure space $(\Gamma, \omega)$ if $\lambda_{f}(\sigma)=\lambda_{g}(\sigma), \sigma>0$, where $\lambda_{f}(\sigma)=\mu\{x:|f(x)|>\sigma\}$ and $\lambda_{g}(\sigma)=\omega\{z:|g(z)|>\sigma\}$. In this sense a function $f(x, y)$ and its rearrangement functions $f^{*}(s, y)$ and $f^{*}(s, t)$ are equimeasurable with respect to their product measures. M. Milman suggested the shortened proof of the following.

THEOREM 2.4. I. Let $g=f^{*}(s, y)$ and $f^{*}(s, t)$ be rearrangement functions for the function $f(x, y)$. If $\sigma>0$ and

$$
\begin{aligned}
\lambda_{g}(\sigma) & =\left|\left\{(s, y) \in[0, \infty) \times \Omega_{2}: g(s, y)>\sigma\right\}\right|, \\
\lambda_{f}(\sigma) & =\left|\left\{(x, y) \in \Omega_{1} \times \Omega_{2}:|f(x, y)|>\sigma\right\}\right|, \\
\lambda_{f^{*}}(\sigma) & =\left|\left\{(s, t) \in[0, \infty) \times[0, \infty): f^{*}(s, t)>\sigma\right\}\right|,
\end{aligned}
$$

then $\lambda_{f}(\sigma)=\lambda_{g}(\sigma)=\lambda_{f *}(\sigma)$.

II. Moreover,

$$
f_{r}^{* *}(s, t) \leqslant\left\{\frac{1}{s t} \int_{0}^{s t} f^{* r}(u) d u\right\}^{1 / r}, \quad 0<r<\infty,
$$

where $f^{*}(u), u>0$, is the one-dimensional rearrangement of the function $f(x, y)$.

Proof. For the proof of $I$ it is enough to show that $\lambda_{f}(\sigma)=\lambda_{g}(\sigma)$. Put $E=$ $\{(x, y):|f(x, y)|>\sigma\}$ and $E^{*}=\{(s, y): g(s, y)>\sigma\}$. For $y$ in $\Omega_{2}$ put $E_{y}=\{x \in$ $\left.\Omega_{1}:(x, y) \in E\right\}$ and $E_{y}^{*}=\left\{s \in[0, \infty):(s, y) \in E^{*}\right\}$, By Lemma 2.1.III, $\mu\left(E_{y}\right)=$ $m\left(E_{y}^{*}\right)$, where $m$ is Lebesgue measure on $[0, \infty)$. We have

$$
(\mu \times \nu)(E)=\int_{\Omega_{2}} \mu\left(E_{y}\right) d y=\int_{\Omega_{2}} m\left(E_{y}^{*}\right) d y=(m \times \nu)\left(E^{*}\right) .
$$

Part II. The corresponding single variable statement of Lemma 2.1.I implies

$$
f_{r}^{* *}(s, t) \leqslant\left\{\frac{1}{s t} \int_{0}^{s t}[f(\cdot, \cdot)]^{* r}(u) d u\right\}^{1 / r}
$$

Part I above implies that $\left[f^{*}(\cdot, \cdot)\right]^{*}(u)=f^{*}(u), u>0$, since two functions which are equimeasurable have the same rearrangement function on $[0, \infty)$. This proves the theorem.

2.2. Simple functions. By an ordinary simple function we mean a function $f(x, y)$ which can be put in the form

$$
f(x, y)=\sum_{k=1}^{N} C_{k} \chi_{E_{k}}(x, y),
$$

where the coefficients are complex valued, $\chi_{E}$ is the characteristic function of the set $E$, the sets $E_{k}$ are disjoint, measurable and contained in a common rectangle $I$ 
of finite measure (here $I$ is of the form $E \times F$, where $E \subset \Omega_{1}, F \subset \Omega_{2}$ and $|E|<\infty,|F|<\infty)$.

By a rectangular simple function is meant an ordinary simple function which can be written in the form

$$
f(x, y)=\sum_{i, j=1}^{N, M} C(i, j) \chi_{E_{i}}(x) \chi_{F_{j}}(y),
$$

where $E_{i} \subset \Omega_{1},\left|E_{i}\right|<\infty, F_{j} \subset \Omega_{2},\left|F_{j}\right|<\infty, i=1, \ldots, N, j=1, \ldots, M$.

For rectangular simple functions some of the coefficients may equal zero. The sets over which these functions are defined are assumed to have coordinate projections which are nonoverlapping. They may be viewed as disjoint rectangles in $\Omega_{1} \times \Omega_{2}$.

It is not hard to show, see [9]: given a measurable function $f(x, y)$ defined on product space $\Omega_{1} \times \Omega_{2}$, then there is a sequence of ordinary simple functions $f_{k}(x, y)$ for which $f_{k} \rightarrow f$ and $\left|f_{k}\right| \uparrow|f|$ pointwise. Here then, Lemma 2.3 applies.

As for rectangular simple functions, let $f(x, y)=\Sigma C_{k} \chi_{E_{k}}$ be an ordinary simple function. By the properties of the product space $\Omega_{1} \times \Omega_{2}$, [9, pp. 137-140]

$$
E_{k}=\bigcap_{j=1}^{\infty} E(k, j), \quad E(k, j)=\bigcup_{p=1}^{\infty} E(k, j, p), \quad k=1, \ldots, N,
$$

where each $E(k, j, p)$ is a rectangle and $I \supset E(k, j, p)$. By working with sets of the form $E(k, J, P)=\cap_{j=1}^{J} \cup_{p=1}^{P} E(k, j, p)$ and using the ring properties of the collection of rectangles in $\Omega_{1} \times \Omega_{2}$, it is a straightforward process to construct a sequence of rectangular simple functions $f_{n}$ such that $f_{n} \rightarrow f$ pointwise a.e. This can be done in such a way that the functions $f_{n}, f$ are uniformly bounded on the rectangle $I$. This discussion shows that rectangular simple functions are useful in context with those function spaces where a Lebesgue dominated convergence theorem holds.

If $f(x, y)=\Sigma C_{k} \chi_{E_{k}}$ is an ordinary simple function with its coefficients rearranged so that $\left|C_{1}\right| \geqslant\left|C_{2}\right| \geqslant \cdots \geqslant\left|C_{N}\right|$, then

$$
f^{*}(u)=\sum_{k=1}^{N}\left|C_{k}\right| \chi_{E_{k}^{*}}(u), \quad u>0,
$$

where $E_{k}^{*}$ is the interval $\left[\sum_{i=1}^{k-1}\left|E_{i}\right|, \sum_{i=1}^{k}\left|E_{i}\right|\right), k=2, \ldots, N$, and $E_{1}^{*}=\left[0,\left|E_{1}\right|\right)$. A corresponding multivariate representation requires a definition and a little more effort.

DEFINITION 2.5. By a rearrangeable simple function we mean a rectangular simple function $f(x, y)$ for which

I. The values $|C(i, j)|$ are all nonzero and distinct.

II. $\left|E_{i}\right|=\alpha,\left|F_{j}\right|=\beta$, some $\alpha>0, \beta>0$ and for each $i, j$.

The assumptions of Definition 2.5 are reasonable. The measure spaces considered are nonnegative, separable and $\sigma$-finite. They are assumed to be either nonatomic or purely atomic with each of the atoms having equal measure. If the spaces $\Omega_{1}$ and $\Omega_{2}$ are purely atomic then condition II is already satisfied. For the nonatomic case and without loss of generality assume that both $\Omega_{1}$ and $\Omega_{2}$ are 
nonatomic. Let $f(x, y)$ be a rectangular simple function all of whose underlying sets have rational measure. There are a fixed number of corresponding underlying sets from the measure spaces $\left(\Omega_{1}, \mu\right)$ and $\left(\Omega_{2}, \nu\right)$. These can be subdivided into a finite number of sets with all of the subsets of $\Omega_{1}$ having equal measure and similarly for the subsets of $\Omega_{2}$. This done, such a function $f(x, y)$ can then be expanded so that the resulting function is again a rectangular simple function which satisfies condition II of Definition 2.5. In those instances where duplications occur with the coefficients it is possible to approximate the coefficients with coefficients uniformly close to the given coefficients but all of distinct nonzero absolute value. This includes those case where $C(i, j)=0$. The result is a rearrangeable simple function. By working with expanding rectangles having sides with rational measure, any rectangular simple function can then be obtained as a pointwise limit of a sequence of such functions (and with the uniformity properties obtained earlier with convergence between the ordinary and rectangular simple functions).

Requiring that the coefficients of rearrangeable simple functions be distinct and nonzero could be omitted. The advantages of these restrictions are purely of a technical nature and lead to the rather nice form of the representation Theorem 2.6 which follows. They allow the coefficients and underlying sets to be easily traced and uniquely indexed. This facilitates any computations made with these functions. Beyond this, the uniqueness property will be required for the proof of one of our main results, Theorem 5.1.

Though we do not consider the topic here it is useful to note that Theorem 2.6 shows that rearrangement simple functions lend themselves naturally to a study of rearrangements and multi-indexed sequence spaces. We state our theorem.

THEOREM 2.6. Let $f(x, y)$ be a rearrangeable simple function as in Definition 2.5, then

$$
f(x, y)=e^{i \arg f(x, y)} \sum_{n, m=1}^{N, M} K(n, m) \chi_{B(n, m)}(x, y)
$$

where $B(n, m)=\{(x, y):|f(x, y)|=K(n, m)\}$ is exactly one of the sets of the form $E_{i} \times F_{j} \subset \Omega_{1} \times \Omega_{2}, 1<i \leqslant N, 1 \leqslant j<M$, without repetitions or omissions. Correspondingly $K(n, m)=C(i, j)$. The coefficients $K(n, m)$ are positive and decreasing for increasing choices of either of the indices $n, m$, where $n=1, \ldots, N, m=1, \ldots, M$. Moreover,

$$
f^{*}(s, t)=\sum_{n, m=1}^{N, M} K(n, m) \chi_{B^{*}(n, m)}(s, t),
$$

where $B^{*}(n, m)=[\alpha(n-1), \alpha n) \times[\beta(m-1), \beta m)$.

Proof. Let $f(x, y)$ be a rearrangeable simple function; then

$$
f(x, y)=\sum_{j}\left(\sum_{i=1}^{N}|C(i, j)| \chi_{E_{i}}(x)\right) \chi_{F_{j}}(y) .
$$

For a given choice of $y$, exactly one of the characteristic functions $x_{F}(y), F=F_{j}$, is nonzero. After rearranging and putting the terms in decreasing order we obtain 
$|f(x, y)|=\sum_{n=1}^{N} K(n, j) \chi_{E(n, j)}(x)$, where $E(n, j)=E_{i^{\prime}}$, some $i^{\prime}, i^{\prime}=1, \ldots, N$, with each set $E_{i}$ appearing exactly once in the rearrangement. $E(n, j)$ depends upon which of the terms $\chi_{F}(y) \neq 0, F=F_{j}$. The relation between $n$ and $i^{\prime}$ is determined by a permutation of the numbers $(1, \ldots, N)$ and is indexed by the indices $j$, $1 \leqslant j \leqslant M$. It is not difficult to see

$$
f^{*}(s, y)=\sum_{n, j=1}^{N, M} K(n, j) \chi_{E^{*}(n, j)}(s) \chi_{F_{j}}(y),
$$

where $E^{*}(n, j)$ is the interval $[\alpha(n-1), \alpha n)$.

The function $f^{*}(s, y)$ has the same basic form as the function $f(x, y)$, with the intervals $[\alpha(n-1), \alpha n)$ playing the role of the sets $E_{i}$ and the variable $s$ replacing the variable $x$. The same construction can then be repeated with the function $f^{*}(s, y)$ and with respect to the variable $y$. Since $f^{*}(s, t)=\left[f^{*}(s, \cdot)\right]^{*}(t)$, the functions $f^{*}(s, t)$ and $f^{*}(s, y)$ in rearranged form, their coefficients, underlying sets and indices are related to each other similarly as are the functions $f(x, y)$ and $f^{*}(s, y)$. Moreover, because the process is iterative, the rearrangement of the terms of $f^{*}(s, y)$ are reflected in kind by a rearrangement of the terms of $f(x, y)$. Following on these remarks the proof can be completed in a straightforward fashion.

\section{Banach function spaces and mixed norms.}

3.1. Banach function spaces. The purpose of this section is to use multivariate rearrangement and averaging functions to define Banach function spaces with mixed norm. In order to do this it is necessary to begin with a short discussion of Banach function spaces. These spaces were introduced by Luxemburg [12], [13]. The presentation is drawn directly from these references.

We consider normed (quasi-normed) function spaces $X=X(\Omega, \mu)$ with norm (quasi-norm) $\|f\|_{X}$, for functions $f(x)$ which are measurable. The measure space $(\Omega, \mu)$ is either nonatomic or discrete (purely atomic with a countable number of atoms of equal measure) and the function space $X$ is rearrangment invariant. That is, if $f(x)$ is equimeasurable with $g(x)$, then $\|f\|_{X}$ is equivalent to $\|g\|_{X}$. Such a space is a Banach function space (with Fatou norm) if:

I. $|f|<|g|$ a.e. $x, g \in X$, implies $f \in X$ and $\|f\|_{X}<\|g\|_{X}$.

II. $\chi_{E} \in X$ whenever $\chi_{E}$ is the characteristic function of a set of finite measure (if $\Omega$ is a product space we require $E \subset I$, for some rectangle $I$ of finite measure).

III. $f \in X$ implies $f$ is locally integrable ( $f \chi_{I} \in X$ if $\Omega$ is a product space).

IV. If $0 \leqslant f_{k} \uparrow f$ pointwise, then $\left\|f_{k}\right\|_{X} \uparrow\|f\|_{X}$ as $k \rightarrow \infty$.

These spaces are norm complete. A Banach function space has absolutely continuous norm if for each $f \in X$ and every sequence of measurable sets $E_{k}$ for which $E_{k} \downarrow \Phi$, then $\left\|f \chi_{E}\right\|_{X} \rightarrow 0, E=E_{k}$, as $k$ tends to infinity. This condition implies that a Lebesgue dominated convergence theorem holds. Namely, if $f_{k} \rightarrow f$ pointwise a.e. and $\left|f_{k}\right| \leqslant|g|$ for some $g$ in $X$, then $\left\|f_{k}-f\right\|_{X} \rightarrow 0$, as $k \rightarrow \infty$. It follows from the discussion in $\$ 2$ that the simple functions are dense in such spaces.

Luxemburg [13] showed that for each rearrangement invariant Banach function space (as described here) there exists a corresponding rearrangement invariant 
Banach function space $X(*)$ defined for all real valued Lebesgue measurable functions on the interval $(0, \mu(\Omega))$ with the property $\|f\|_{X}=\left\|f^{*}\right\|_{X(*)}$. For convenience and when there is not danger of ambiguity we will sometimes put $\left\|f^{*}\right\|_{X}=$ $\left\|f^{*}\right\|_{X(*)}$. Here $f^{*}(s)$ represents the one variable rearrangement of the function $f(x)$.

Let $f(x)$ be a measurable function. The first and second associated norms of $f(x)$ are defined by

$$
\begin{aligned}
& \|f\|_{X^{\prime}}=\sup \left\{\int|f g| d u:\|g\|_{X}<1\right\}, \\
& \|f\|_{X^{\prime \prime}}=\sup \left\{\int|f g| d u:\|g\|_{X^{\prime}}<1\right\}
\end{aligned}
$$

with $X^{\prime}$ and $X^{\prime \prime}$ being the corresponding function spaces of all measurable $f(x)$ with finite norm. These spaces are also rearrangement invariant Banach function spaces. Moreover,

$$
\begin{aligned}
\int|f g| d u & \leqslant\|f\|_{X}\|g\|_{X^{\prime}} \quad \text { (Hölder's inequality), } \\
\|f\|_{X^{\prime}} & =\sup \left\{\int_{0}^{|\Omega|} f^{*}(s) g^{*}(s) d s:\|g\|_{X}<1\right\}, \\
\|f\|_{X^{\prime \prime}} & =\sup \left\{\int_{0}^{|\Omega|} f^{*}(s) g^{*}(s) d s:\|g\|_{X^{\prime}}<1\right\} .
\end{aligned}
$$

If $|\Omega|$ is finite then $L^{\infty} \subset X \subset L^{1}$, continuously. $X^{\prime}$ is a closed subspace of $X^{*}$, the Banach dual of the space $X$. If $X$ has absolutely continuous norm, then $X^{\prime}=X^{*}$. A deep result of Lorentz and Luxemburg [13] is that $\|f\|_{X^{\prime \prime}}=\|f\|_{X}$.

By using the properties of $X$ and its associated spaces it is not hard to show that a Minkowski integral inequality holds. Namely, if $f(x, y) \in X$ for each $y$ and $\|f(\cdot, y)\|_{X} \in L^{r}, 0 \leqslant r \leqslant 1$, where $L^{r}$ is classical Lebesgue space, then

$$
\|\| f\left\|_{L^{\prime}}\right\|_{X} \leqslant\|\| f\left\|_{X}\right\|_{L^{r}}
$$

We remark that some well-known examples to which these discussions apply are: the classical $L^{p}$ spaces, the Orlicz classes $\Lambda\left(\gamma_{X}, C\right)$ [21], the Lorentz-Zygmund spaces $L^{p a}(\log L)^{\alpha}[2]$, the Lorentz $\Lambda_{\alpha}(X)$ spaces [19] and the Lorentz $L(p, q)$ spaces [11]. For certain choices of say the underlying Young's function, fundamental function or certain choices of the indices, several of these function spaces satisfy Hardy type inequalities. In these cases,

$$
\left\|f^{*}\right\|_{X} \leqslant\left\|f_{r}^{* *}\right\|_{X} \leqslant C\left\|f^{*}\right\|_{X^{\prime}}
$$

for some $r, 0<r<\infty$ (may choose $0<r \leqslant 1$ ) and some constant $C$ independent of the function $f(x)$.

3.2. Mixed norm spaces. Let $X=X\left(\Omega_{1}, \mu\right)$ and $Y=Y\left(\Omega_{2}, \nu\right)$ be rearrangement invariant Banach function spaces with corresponding Luxemburg representations $X(*)$ and $Y(*)$. As before, let $f(x, y)$ be a measurable function defined on the product space $\left(\Omega_{1} \times \Omega_{2}, \mu \times \nu\right)$. The mixed norm (quasi-norm) space $Y[X]$, in the sense of Benedek and Panzone [1], is:

$$
Y[X]=\left\{f:\|f\|_{Y[X]}=\|\| f(\cdot, y)\left\|_{X}\right\|_{Y}<\infty\right\} .
$$


Using Luxemburg representations,

$$
\|f\|_{Y[X]}=\left\|\left(\left\|f^{*}(\cdot, y)\right\|_{X(*)}\right) *(t)\right\|_{Y(*)} .
$$

We denote the space $Y[X](*)$ by

$$
Y[X](*)=\left\{f:\|f\|_{Y[X](*)}=\left\|f^{*}(s, t)\right\|_{Y[X]}=\|\| f^{*}(s, t)\left\|_{X(*)}\right\|_{Y(*)}<\infty\right\} .
$$

Using iteration and the remarks made so far it is not difficult to verify that both $Y[X]$ and $Y[X](*)$ are Banach function spaces, though not necessarily rearrangement invariant. We omit the details. Moreover, if $X$ and $Y$ have absolutely continuous norms, then so do the spaces $Y[X]$ and $Y[X](*)$. Since these spaces in turn satisfy the Lebesgue Dominated Convergence Theorem, it follows that the ordinary, rectangular and rearrangeable simple functions are dense in such spaces.

If $X$ and $Y$ satisfy the Hardy inequalities, then Lemma 2.2.III, (3.6) and (3.7) imply

$$
\left\|f^{*}\right\|_{Y[X]} \leqslant\left\|\bar{f}_{r}\right\|_{Y[X]} \leqslant\left\|f_{r}^{* *}\right\|_{Y[X]} \leqslant C\left\|f^{*}\right\|_{Y[X]},
$$

some $r, 0<r<\infty$ (may choose $0<r \leqslant 1$ ) and some constant $C$ independent of the function $f(x, y)$.

The corresponding associated spaces of $Y[X]$ and $Y[X](*)$ are denoted by $(Y[X])^{\prime},(Y[X])^{\prime \prime}$ and $(Y[X](*))^{\prime},(Y[X](*))^{\prime \prime}$ respectively. The associated spaces of $X$ and $Y$ with mixed norm are defined to be $Y^{\prime}\left[X^{\prime}\right], Y^{\prime \prime}\left[X^{\prime \prime}\right]$ and $Y^{\prime}\left[X^{\prime}\right](*)$, $Y^{\prime \prime}\left[X^{\prime \prime}\right](*)$.

For two functions $f(x, y)$ and $g(x, y)$, Lemma 2.1.II and Theorem 2.4.II imply

$$
\begin{aligned}
\iint|f(x, y) g(x, y)| d x d y & \leqslant \int_{0}^{\infty} \int_{0}^{\infty} f^{*}(s, t) g^{*}(s, t) d s d t \\
& \leqslant \int_{0}^{\infty} f^{*}(u) g^{*}(u) d u,
\end{aligned}
$$

where $f^{*}(u)$ and $g^{*}(u)$ are the one variable rearrangements of $f$ and $g$. This in turn readily leads to a wealth of relationships between $X, Y$ and the various associated spaces defined above. For the sake of brevity we only list four of the most important.

TheOREM 3.12. I. $(Y[X])^{\prime}=Y^{\prime}\left[X^{\prime}\right]$.

II. $(Y[X](*))^{\prime}=Y^{\prime}\left[X^{\prime}\right](*)$.

If $X$ and $Y$ have absolutely continuous norms, then

III. $(Y[X])^{\prime}=(Y[X])^{*}=Y^{*}\left[X^{*}\right]$.

IV. $(Y[X](*))^{\prime}=(Y[X](*))^{*}=Y^{*}\left[X^{*}\right](*)$

where $X^{*}$ and $Y^{*}$ are the Banach dual spaces of $X$ and $Y$ respectively.

Proof. The proof of III and IV follow immediately from I, II and the previous discussions. We prove II; the proof of $I$ is similar. Inclusion of $Y^{\prime}\left[X^{\prime}\right](*)$ follows easily by (3.11) and successive applications of Hölder's inequality. For the reverse inclusions let $f(x, y) \in(Y[X](*))^{\prime}$ and let $I=I_{1} \times I_{2}$ be a rectangle in $\Omega_{1} \times \Omega_{2}$ 
with finite measure; then

$$
\|\|\left(f \chi_{I}\right)^{*}\left\|_{X^{\prime}}\right\|_{Y^{\prime}}=\sup _{h} \int_{0}^{\left|I_{2}\right|} \sup _{g} \int_{0}^{\left|I_{1}\right|}\left(f \chi_{I}\right)^{*}(s, t) g(s, t) d s h(t) d t,
$$

where $\left\|g^{*}(\cdot, t)\right\|_{X(*)} \leqslant 1,\left\|h^{*}\right\|_{Y(*)} \leqslant 1$ and whose support is contained in the rectangle $\left[0,\left|I_{1}\right|\right) \times\left[0,\left|I_{2}\right|\right)$. For each $\varepsilon>0$ there exist measurable functions $g(s, t)$ and $h(t)$ as above so that the quantity on the right is majorized by

$$
\int_{0}^{\left|I_{2}\right|} \int^{\left|I_{1}\right|}\left(f \chi_{I}\right)^{*}(s, t) g^{*}(s, t) h^{*}(t) d s d t+\int_{0}^{\left|I_{2}\right|} \varepsilon h^{*}(t) d t+\varepsilon
$$

Since $\left\|g^{*}(s, t) h^{*}(t)\right\|_{\left.Y[X\}_{*}\right)} \leqslant 1$, this is majorized by

$$
\sup _{k} \int_{0}^{\infty} \int_{0}^{\infty}\left(f \chi_{I}\right)^{*}(s, t) k(s, t) d s d t+\varepsilon\left\|h^{*} \chi_{\left[0,\left|I_{1}\right|\right)}\right\|_{L^{1}}+\varepsilon .
$$

Since $L_{Y(*)}\left(0,\left|I_{2}\right|\right) \subset L^{1}\left(0,\left|I_{2}\right|\right)$, this is majorized by

$$
\left\|\left(f \chi_{I}\right)^{*}\right\|_{(Y[X])^{\prime}}+\varepsilon K\left\|h^{*}\right\|_{Y(*)} \text {. }
$$

This in turn is less than or equal to $\left\|f^{*}\right\|_{(Y[X])^{\prime}}+\varepsilon K+\varepsilon$, where the constant $K$ depends only on $Y$ and the rectangle $I$. By letting $\varepsilon \rightarrow 0$ followed by letting $I \uparrow \Omega_{1} \times \Omega_{2}$ on the left, we obtain $\left\|f^{*}\right\|_{Y^{\prime}\left[X^{\prime}\right]} \leqslant\left\|f^{*}\right\|_{\left(Y[X]^{\prime}\right.}$. This completes the proof.

3.3. Inclusions. Let $X$ and $Y$ be rearrangement invariant Banach function spaces. Let $X^{\prime}$ be the first associated space of $X$. If $X$ has absolutely continuous norm then $X^{\prime}=X^{*}$. A natural context for the spaces $Y[X]$ and $Y[X](*)$ are as intermediate spaces between the function space $I\left(X^{\prime}, Y\right)$ consisting of kernels of integral operators which are bounded from $X^{\prime}$ to $Y$ and the function space $X \otimes_{\gamma} Y$, which is the completion of the algebraic tensor product of $X$ and $Y$ in the greatest cross norm $\gamma$. For a more detailed treatment of integral operators and tensor products see [14], [17], [18]. The statement of this is formally given in

THEOREM 3.13. With continuous embeddings,

I. $X \otimes_{\gamma} Y \subset Y[X] \subset I\left(X^{\prime}, Y\right)$.

II. $X \otimes_{\gamma} Y \subset Y[X](*) \subset I\left(X^{\prime}, Y\right)$.

Proof. We prove only II; the proof of $I$ is well known and done similarly. Recall that if $k(x, y) \in I\left(X^{\prime}, Y\right)$, then an operator $T_{k}: X^{\prime} \rightarrow Y$ is defined by

$$
T_{k} f(y)=\int_{\Omega_{1}} k(x, y) f(x) d x, \quad f \in X^{\prime} .
$$

The norm of the operator is $\left\|T_{k}\right\|$ which is the supremum of al values $\left\|T_{k} f\right\|_{Y}$ for which $\|f\|_{X^{\prime}}=\left\|f^{*}\right\|_{X^{\prime}(*)} \leqslant 1$. For such a function $f(x),\left\|T_{k} f\right\|_{Y}=$ $\left\|\int_{\Omega_{1}} k(x, y) f(x) d x\right\|_{Y}$. Lemma 2.1.II and (3.2) imply this is majorized in order by

$$
\begin{aligned}
\left\|\int_{0}^{\infty} k^{*}(s, y) f^{*}(s) d s\right\|_{Y} & =\sup _{g} \int_{\Omega_{2}} \int_{0}^{\infty} k^{*}(s, y) f^{*}(s) d s g(y) d y . \\
& \leqslant \sup _{g} \int_{0}^{\infty} \int_{0}^{\infty} k^{*}(s, t) f^{*}(s) g^{*}(t) d s d t,
\end{aligned}
$$

where $\|g\|_{X^{\prime}}=\left\|g^{*}\right\|_{X^{\prime}} \leqslant 1$. Since $\left\|f^{*}(s) g^{*}(t)\right\|_{Y^{\prime}\left[X^{\prime}\right\}(*)}<1$, Hölder's inequality implies $\left\|T_{k} f\right\|_{Y} \leqslant\left\|k^{*}\right\|_{Y[X](*)}$. This proves the second inclusion. 
For the first inclusion, let $h(x, y)$ be a function which can be represented by a terminating sum of the form $h(x, y)=\sum f_{k}(x) g_{k}(y)$, for which $\|h\|_{X \otimes_{\gamma} Y}=$ inf $\Sigma\left\|f_{k}\right\|_{X}\left\|g_{k}\right\|_{Y}$ is finite, where the infimum is over all such representations for $h(x, y)$. It is not difficult to see that for each such representation, $\bar{h}(s, t)<$ $\sum \bar{f}_{k}(s) \bar{g}_{k}(t)$ and $\|\bar{h}\|_{Y[X](*)} \leqslant \sum\left\|\bar{f}_{k}\right\|_{X(*)}\left\|\bar{g}_{k}\right\|_{Y(*)}$. If the Hardy inequalities hold for $X$ and $Y$, then $\left\|h^{*}\right\|_{Y[X]} \leqslant C\|h\|_{X \otimes_{Y} Y}$. This implies $Y[X](*) \supset X \otimes_{Y} Y$.

If $X$ and $Y$ are rearrangement invariant Banach function spaces defined on $[0, \infty)$ and $Y[X]$ is rearrangement invariant, it is immediate that $Y[X]=Y[X](*)$. In the next section we show for certain function spaces $X, Y$ where $Y[X]$ is not necessarily rearrangement invariant that containment relationships can exist between $Y[X]$ and $Y[X](*)$. Moreover, if $X=L^{p}[0, \infty), 1 \leqslant p<\infty$, and $Y=$ $L^{\infty}[0, \infty)$, then using Theorem 4.5 and an example of the form $f(x, y)=y$, $0<x y^{p} \leqslant 1$ and zero otherwise, shows that containment can be proper.

REMARK. The results obtained so far carry through to product spaces of dimension $n, n \geqslant 2$. If $(\Omega, \mu)=\left(\times_{i=1}^{n} \Omega_{i}, X_{i=1}^{n} \mu_{i}\right)$ is such a space, then let $f(x)$, $x=\left(x_{1}, \ldots, x_{n}\right)$, be a complex valued measurabe function defined on $(\Omega, \mu)$. By iteration we define the nonnegative, nonincreasing rearrangement of $f(x)$, up to $k$, $1 \leqslant k \leqslant n$ and in the order $(1, \ldots, k)$, to be

$$
f^{*}\left(t_{1}, \ldots, t_{k}, x_{k+1}, \ldots, x_{n}\right)=\left[\cdots\left[f^{*}\left(t_{1}, \cdot\right)\right]^{*}\left(t_{2}, \cdot\right) \cdots\right]^{*}\left(t_{k}, x_{k+1}, \ldots, x_{n}\right) \text {, }
$$

where $t_{i}>0 ; i=1, \ldots, k$, and $f^{*}(t)=f^{*}\left(t_{1}, \ldots, t_{n}\right)$. The averaging operators $\bar{f}_{r}\left(t_{1}, \ldots, t_{k}, x_{k+1}, \ldots, x_{n}\right)$ and $\bar{f}_{r}(t)$ are defined similarly. The averaging operators $f_{r}^{* *}$ are also defined naturally. And explicitly

$$
f_{r}^{* *}(t)=\left[\left(t \cdots t_{n}\right)^{-1} \int_{0}^{t_{n}} \cdots \int_{0}^{t_{1}} f^{* r}\left(u_{1}, \ldots, u_{n}\right) d u_{1}, \ldots, d u_{n}\right]^{1 / r}
$$

The natural order of indexing $(1, \ldots, k)$ was picked above. This will be sufficient for our purposes. Continuing, let $X_{i}$ be rearrangement invariant Banach function spaces defined on the measure spaces $\left(\Omega_{i}, \mu_{i}\right)$ and with corresponding Luxemburg representations $X_{i}(*) ; i, \ldots, n$. The mixed norm space $\underline{X}$ and the space $\underline{X}(*)$ are defined in the natural way by putting $\underline{X}=X_{n}\left[\cdots\left[X_{2}\left[X_{1}\right]\right] \cdots\right]$ and $\underline{X}(*)=X_{n}\left[\cdots\left[X_{2}\left[X_{1}\right]\right] \cdots\right](*)$.

Throughout the rest of our discussions we will assume that the results of $\S \S 2$ and 3 have been extended to the multivariate case $n, n \geq 2$. Doing this is a straightforward process and for the most part simple induction suffices. Verification of the details is left to the discretion of the reader.

4. Lorentz spaces $L(P, Q ; *)$.

4.1. The remainder of the paper will be devoted to a discussion of the Lorentz $L(p, q)$ spaces and mixed norms. These function spaces are one of the main topics of the paper and a main application of the ideas presented so far. Let $f(x)$, $x=\left(x_{1}, \ldots, x_{n}\right)$, be measurable and finite a.e. and let $f^{*}(t), t=\left(t_{1}, \ldots, t_{n}\right)$ be its nonincreasing rearrangement. Let $L\left(p_{1}, q_{1}\right), \ldots, L\left(p_{n}, q_{n}\right)$ be Lorentz $L(p, q)$ spaces with quasi-norms $\left\|g^{*}\right\|_{p q^{*}}^{*}$; see [11]. The Lorentz mixed norm space $L(P, Q)$ and the Lorentz space $L(P, Q: *)$ are defined by 


$$
\begin{aligned}
L(P, Q) & =L\left(p_{n}, q_{n}\right)\left[\cdots\left[L\left(p_{2}, q_{2}\right)\left[L\left(p_{1}, q_{1}\right)\right]\right] \cdots\right], \\
L(P, Q ; *) & =L\left(p_{n}, q_{n}\right)\left[\cdots\left[L\left(P_{2}, q_{2}\right)\left[L\left(p_{1}, q_{1}\right)\right]\right] \cdots\right](*)
\end{aligned}
$$

respectively, where $P=\left(p_{1}, \ldots, p_{n}\right), Q=\left(q_{1}, \ldots, q_{n}\right)$ and the quasi-norms are denoted by $\|f\|_{P Q}^{*}=\|f\|_{L(P, Q)}^{*}$ and $\left\|f^{*}\right\|_{P Q}^{*}=\left\|f^{*}\right\|_{L(P, Q ; *}^{*}$.

Throughout the paper the letters $P, Q, \ldots, R$ will denote $n$-tuples, $n>1$, $P=\left(p_{1}, \ldots, p_{n}\right), \ldots, R=\left(r_{1}, \ldots, r_{n}\right)$. Moreover, if $\Phi(p, q, \ldots, r)$ is a relation among numbers, $\Phi(P, Q, \ldots, R)$ will mean $\Phi\left(p_{i}, q_{i}, \ldots, r_{i}\right)$ holds for each $i=$ $1, \ldots, n$. An example is: if $p^{\prime}=p /(p-1)$ is the conjugate value for $p>1$, then $P^{\prime}(P-1)$ is the $n$-tuple whose components are the conjugate values of the components of $P$.

In previous sections the discussions were presented primarily in terms of the $n$-dimensional case $n=1$ or $n=2$. Here, for the sake of future reference and because it is no more difficult, the discussions will be in the context of dimension $n$, $n \geqslant 1$. Also, in subsequent sections we present our main results and some of their applications. These will be given for $L(P, Q ; *)$ spaces. For this reason the discussions will be restricted primarily to these function spaces. The reader should note however, in view of the previous discussions in $\$ \S 2$ and 3 , that several of the statements of this particular section carry over in a straightforward fashion to the $L(P, Q)$ spaces.

Following directly in the discussions of $\$ 3$ and the known properties of the $L(p, q)$ spaces: If $1 \leqslant p<\infty, 1 \leqslant Q \leqslant \infty$, then $L(P, Q ; *)$ with quasi-norm $\left\|f^{*}\right\|_{P Q}^{*}$ is a Banach function space. Here it is required: if $p_{i}=1$ some $i$, then $q_{i}=1$ or if $p_{i}=\infty$ some $i$, then $q_{i}=\infty, i=1, \ldots, n$. Those spaces where $1<p<\infty$, $1 \leqslant Q<\infty$, have absolutely continuous norm, which implies $L(P, Q ; *)=$ $L\left(P^{\prime}, Q^{\prime} ; *\right)$, where $1 / P+1 / P^{\prime}=1$ and $1 / Q+1 / Q^{\prime}=1$. In addition, the set of ordinary, rectangular and rearrangeable simple functions are dense for this particular choice of indices. If $q_{i}=\infty$, some $i, i=1, \ldots, n$, then as with the one-dimensional case $L(p, q)$, the dual of $L(P, Q ; *)$ cannot be expected to be $L\left(P^{\prime}, Q^{\prime} ; *\right)$ and the collection of simple functions do not form a dense subset.

Hölder's inequality takes the form

$$
\begin{aligned}
\int|f(x) g(x)| d x & \leqslant \int_{0}^{\infty} \cdots \int_{0}^{\infty} f^{*}\left(t_{1}, \ldots, t_{n}\right) g^{*}\left(t_{1}, \ldots, t_{n}\right) d t_{1} \cdots d t_{n} \\
& \leqslant\left\|f^{*}\right\|_{P Q}^{*}\left\|g^{*}\right\|_{P^{\prime} Q^{\prime}}^{*}
\end{aligned}
$$

It is worth mentioning that, as in [11], it is possible to consider $L(P, Q ; *)$ spaces for $0<P \leqslant \infty, 0<Q \leqslant \infty$. Choose $r, 0<r<1$, where $r<P, r \leqslant Q$, then it can be shown that $d(f, g)=\|{\overline{(f-g)_{r}}}_{\|_{P Q}^{* r}}$ defines a metric on $L(P, Q ; *)$. Moreover, Lemma 2.2.III, Minkowski's integral inequality and the Hardy inequalities [11] imply

$$
\left\|f^{*}\right\|_{P Q}^{*} \leqslant\left\|\bar{f}_{r}\right\|_{P Q}^{*} \leqslant\left\|f_{r}^{* *}\right\|_{P Q}^{*} \leqslant C\left\|f^{*}\right\|_{P Q}^{*}
$$

where $C$ is independent of the function $f$. Completeness with respect to the metric and density of the simple functions, $0<P<\infty, 0<Q<\infty$ can be shown to follow. 
Lorentz $L(P, Q ; *)$ spaces with different indices are related in some special case. If $0<Q_{1} \leqslant Q_{2} \leqslant \infty$, then

$$
L\left(P, Q_{1} ; *\right) \subset L\left(P, Q_{2} ; *\right) \text {, with }\left\|f^{*}\right\|_{P Q_{2}}^{*} \leqslant C\left\|f^{*}\right\|_{P Q_{1}}^{*},
$$

where $C$ depends only on the indices. If for each $i, \mu_{i}\left(\Omega_{i}\right)<\infty, 0<P_{1}<P_{2}<\infty$, $0<Q_{1}, Q_{2} \leqslant \infty$, then

$$
L\left(P_{2}, Q_{2} ; *\right) \subset L\left(P_{1}, Q_{1} ; *\right), \quad \text { with }\left\|f^{*}\right\|_{P_{1} Q_{1}}^{*}<C\left\|f^{*}\right\|_{P_{2} Q_{2}}^{*},
$$

where $C$ depends only on the indices and the measure space $\Omega$. If $\mu_{i}=\infty$, then (4.4) can be modified in the obvious way by requiring that the $i$ th components of $P_{1}$ and $P_{2}$ be equal and that the $i$ th component of $Q_{2}$ be less than or equal to the $i$ th component of $Q_{1}$. Both (4.3) and (4.4) can easily be verified by using iteration and the one variable proofs given in [11]. The details are omitted.

The spaces most closely related to the $L(P, Q ; *)$ spaces are the Lorentz $L(p, q)$ spaces and the Lorentz mixed norm spaces $L(P, Q)$. The following theorem provides containment relationships between these various function spaces.

TheOREM 4.5. I. If $P=\left(p_{1}, \ldots, p_{n}\right), Q=\left(q_{1}, \ldots, q_{n}\right) ; 1<P<\infty, 1<Q<$ $\infty$ and

$$
\max _{1<i<n-1}\left\{q_{i}\right\}<\min _{2<j<n}\left\{p_{j}\right\}, \quad 1 / P+1 / P^{\prime}=1,1 / Q+1 / Q^{\prime}=1,
$$

then $L(P, Q ; *) \subset L(P, Q)$ and $L\left(P^{\prime}, Q^{\prime}\right) \subset L\left(P^{\prime}, Q^{\prime} ; *\right)$.

II. Let $P=(p, \ldots, p), Q=(q, \ldots, q)$ and $\bar{Q}=(q, \infty, \ldots, \infty)$. Then $L(p, q)$ $\subset L(P, \bar{Q} ; *)$. If $(\Omega, \mu)$ is Euclidean $n$-space with Lebesgue measure and if $p \neq q$, $q \neq \infty$, then $L(P, Q ; *) \neq L(p, q)$ and neither space contains the other.

III. If $p_{i}=q_{i}=p ; i=1, \ldots, n$, so that $P=Q=(p, \ldots, p)$, then $L^{p}=L^{p}(*)$ $=L(p, p)$ and $L^{P}=L^{P}(*)=L(P, P ; *)=L(P, P)$ with equality of the norms (quasi-norms).

Proof. We prove the left inclusion of part I first and do it by majorization of norms. The proof is by induction on the number of variables. Since $L(P, Q ; *)=$ $L(p, q)$, if $P=p, Q=q$, the case for $n=1$ is obvious. Suppose continuous inclusion has been shown for $n-1, n \geqslant 2$. Then

$$
\|f\|_{L(P, Q)} \leqslant C\|\| \cdots\left\|f^{*}\left(t_{1}, \ldots, t_{n-1}, x_{n}\right)\right\|_{p_{1} q_{1}}^{*} \cdots\left\|_{p_{n-1} q_{n-1}}^{*}\right\|_{L\left(p_{n}, q_{n}\right)}^{*}
$$

where $C$ depends only on $P$ and $Q$. Put

$$
F\left(t_{2}, \ldots, t_{n-1}, x_{n}\right)=\left\|f^{*}\left(\cdot, t_{2}, \ldots, t_{n-1}, x_{n}\right)\right\|_{p_{1} q_{1}}^{*} .
$$

Again by the hypothesis and after noting the function $F$ is already nonincreasing in the variables $t_{2}, \ldots, t_{n-1}$, the last term is majorized by

$$
C\|\cdots\|\left\|F^{*}\left(t_{2}, \ldots, t_{n}\right)\right\|_{p_{2} q_{2}}^{*}\|\cdots\|_{p_{n} q_{n}}^{*} .
$$

Consider the function $F^{*}$,

$$
F^{*}\left(t_{2}, \ldots, t_{n}\right) \leqslant \sup \left[\frac{1}{|E|} \int_{E} F\left(t_{2}, \ldots, t_{n-1}, x_{n}\right)^{r} d x_{n}\right]^{1 / r}
$$

where the supremum is over all sets $E=E\left(t_{n} ; t_{2}, \ldots, t_{n-1}\right),|E| \geqslant t_{n}$. Put $r=q_{1}$ : 
then Fubini's theorem and Lemma 2.1 imply $F^{*}$ is majorized by

$$
\left[\frac{1}{t_{n}} \int_{0}^{t_{n}}\left\|f^{*}\left(\cdot, t_{2}, \ldots, u_{n}\right)\right\|_{p_{1} q_{1}}^{* r} d u_{n}\right]^{1 / r} \text {. }
$$

If $r \geqslant 1$, it is not difficult to show that

$$
f_{r}^{* *}(u) \leqslant 2 u^{-1 / r} \int_{0}^{u} v^{-1 / r^{\prime}} f^{*}(v) d v, \quad 0<u<\infty, 1 / r+1 / r^{\prime}=1 .
$$

This implies the last expression is majorized accordingly with $r=q_{1}$. After subsituting back followed by successive uses of Minkowski's integral inequality,

$$
\|f\|_{L(P, Q)} \leqslant C\left\|t_{n}^{-1 / r} \int_{0}^{t_{n}} u_{n}^{-1 / r^{\prime}}\right\| \cdots\left\|f^{*}\left(\cdot, u_{n}\right)\right\|_{p_{1} q_{1}}^{*} \cdots\left\|_{p_{n-1} q_{n-1}}^{*} d u_{n}\right\|_{p_{n} q_{n}}^{*} .
$$

Since $r=q_{1}<p_{n}$ the conclusion follows by Hardy's inequality [11].

The inclusion on the right in part I follows by a simple duality argument which makes use of Hölder's inequality and the inclusion on the left. We omit the details.

Part II. By Theorem 2.4,

$$
f_{r}^{* *}(t) \leqslant\left[\left(t_{n} \cdots t_{1}\right)^{-1} \int_{0}^{t_{n} \cdots t_{1}} f^{* r}(u) d u\right]^{1 / r}
$$

With a change of variables,

$$
\left\|f_{r}^{* *}\left(\cdot, t_{2}, \ldots, t_{n}\right)\right\|_{p q}^{*} \leqslant\left(t_{n} \cdots t_{2}\right)^{-1 / p}\left\|f_{r}^{* *}(\cdot)\right\|_{L(p, q)}^{*}
$$

This implies $\left\|f_{r}^{* *}(\cdot, \ldots, \cdot)\right\|_{P \bar{Q}}^{*}<\left\|f_{r}^{* *}(\cdot)\right\|_{p q}$ and $L(p, q) \subset L(P, \bar{Q} ; *)$. The second statement of part II follows directly from a lemma given by Cwikel [6]. The result there was given for $L(P, Q)$ spaces. But since his constructions were in terms of multivariate nonincreasing functions, the examples apply equally well to the $L(P, Q ; *)$ spaces.

The second equality on the right of part III can be easily verified by repeated use of Fubini's theorem and Lemma 2.1.I in comparing norms. The other equalities are equally easy to verify. Again, we omit the details. This completes the proof of the theorem.

\section{Applications.}

5.1. An interpolation theorem. In this section we extend the Strong Type (RieszThorin) Theorem to the $L(P, Q ; *)$ spaces. See [11]. The theorem will be given for a sublinear operator $T$ which maps measurable functions on an $n$-dimensional measure space $\times \Omega_{i}$ into measurable functions on an $m$-dimensional measure space $\times \Gamma_{j}$. An operator $T$ is sublinear if whenever $T f$ and $T g$ are defined and $c$ is a constant, then $T(f+g)$ and $T(c f)$ are defined with

$$
|T(f+g)| \leqslant|T f|+|T g| \text { and }|T(c f)| \leqslant|c||T f| \text {. }
$$

THEOREM 5.1. If $T$ is a sublinear operator and $\left\|(T f)^{*}\right\|_{P_{j}^{\prime} Q_{j}^{\prime}}^{*}<C_{j}\left\|f^{*}\right\|_{P_{j} Q_{j}}^{*}, j=0,1$, then

$$
\left\|(T f)^{*}\right\|_{P^{\prime} Q^{\prime}}^{*} \leqslant C C_{0}^{1-\theta} C_{1}^{\theta}\left\|f^{*}\right\|_{P Q}^{*}
$$

where $1 / P=(1-\theta) / P_{0}+\theta / P_{1}, 1 / P^{\prime}=(1-\theta) / P_{0}^{\prime}+\theta / P_{1}^{\prime}, 1 / Q=$ $(1-\theta) / Q_{0}+\theta / Q_{1}, 1 / Q^{\prime}=(1-\theta) / Q_{0}^{\prime}+\theta / Q_{1}^{\prime}, 0<\theta<1$. 
The lengthy proof is deferred to the Appendix 5.4.

5.2. Convolution of functions. Let $\Omega=R^{n}$ be Euclidean $n$-space with Lebesgue measure. The ordinary convolution of two measurable functions $f$ and $g$ defined on $R^{n}$ is given by $(f * g)(y)=\int_{R^{n}} f(x) g(y-x) d x$, whenever the integrals exist and are finite. The authors of [16], [3] and several others have written extensively on operators which are generalizations of ordinary convolution. For the purposes of this paper the discussion is restricted to ordinary convolution. The next lemma extends the basic convolution lemma $[16]$ to the $L(P, Q ; *)$ spaces.

LEMMA 5.2. If $h(y)=(f * g)(y)$, then

$$
\bar{h}(t) \leqslant \int_{t_{n}}^{\infty} \cdots \int_{t_{1}}^{\infty} \bar{f}(u) \bar{g}(u) d u
$$

where $u=\left(u_{1}, \ldots, u_{n}\right), d u=d u_{1} \cdots d u_{n}$

Proof. It is sufficient to consider the case $n=2$. The case for $n>2$ follows by induction. Fix $t_{1}>0, t_{2}>0$ and let $E \subset R, E=E\left(t_{1} ; y_{2}\right),|E|>t_{1}$; then

$$
\frac{1}{|E|} \int_{E} f * g\left(y_{1}, y_{2}\right) d y_{1} \leqslant \int_{R} \frac{1}{|E|} \int_{E} \int_{R}\left|f\left(x_{1}, x_{2}\right) g\left(x_{1}-y_{1}, x_{2}-y_{2}\right)\right| d x_{1} d y_{1} d x_{2} \text {. }
$$

By taking supremums first on the right and then on the left,

$$
\bar{h}\left(t_{1}, y_{2}\right) \leqslant \int_{R}\left(\overline{\int_{R} f\left(x_{1}, x_{2}\right) g\left(\cdot-x_{1}, y_{2}-x_{2}\right) d x_{1}}\right)\left(t_{1}, y_{2}\right) d x_{2} .
$$

By the one variable proof [16],

$$
\leqslant \int_{R} \int_{t_{1}}^{\infty} \bar{f}\left(u_{1}, x_{2}\right) \bar{g}\left(u_{1}, y_{2}-x_{2}\right) d u_{1} d x_{2} \text {. }
$$

The proof is completed by using Fubini's theorem and repeating the procedure above with respect to the second variable.

The convolution theorem is as follows.

TheOREM 5.3. If $h=f * g, f \in L\left(P_{1}, Q_{1} ; *\right)$ and $g \in L\left(P_{2}, Q_{2} ; *\right)$, where $2>$ $1 / P_{1}+1 / P_{2} \geqslant 1,1 \leqslant P_{1}, P_{2}<\infty$, then $h \in L(R, S ; *)$, where $1 / R+1=1 / P_{1}$ $+1 / P_{2}$ and $S \geqslant 1$ is such that $1 / Q_{1}+1 / Q_{2} \geqslant 1 / S$. Moreover,

$$
\left\|h^{*}\right\|_{R S}^{*} \leqslant C\left\|f^{*}\right\|_{P_{1} Q_{1}}^{*}\left\|g^{*}\right\|_{P_{2} Q_{2}}^{*}
$$

where $C$ depends only on the indices.

Proof. As before, it is sufficient to prove the case $\boldsymbol{n}=2$. By Lemmas 2.2.III and $5.2, h^{*}\left(t_{1}, t_{2}\right) \leqslant \int_{t_{2}}^{\infty} \int_{t_{1}}^{\infty} f^{* *}\left(u_{1}, u_{2}\right) g^{* *}\left(u_{1}, u_{2}\right) d u_{1} d u_{2}$. By Minkowski's integral inequality

$$
\left\|h^{*}\left(\cdot, t_{2}\right)\right\|_{r_{1} s_{1}} \leqslant \int_{t_{1}}^{\infty}\left\|\int_{(*)}^{\infty} f^{* *}\left(u_{1}, u_{2}\right) g\left(u_{1}, u_{2}\right) d u_{1}\right\|_{r_{1} s_{1}}^{*} d u_{2} .
$$

By the one variable proof [16],

$$
\leqslant \int_{t_{2}}^{\infty}\left\|f^{* *}\left(\cdot, u_{2}\right)\right\|_{p_{11} q_{11}}\left\|g^{* *}\left(\cdot, u_{2}\right)\right\|_{p_{12} q_{12}} d u_{2} \text {. }
$$

Here, $P_{1}=\left(p_{11}, p_{21}\right), P_{2}=\left(p_{12}, p_{22}\right), Q_{1}=\left(q_{11}, q_{21}\right), Q_{2}=\left(q_{12}, q_{22}\right)$. The proof is 
completed by repeating the same procedure with respect to the second variable.

5.3. Fractional integration. Let $\Omega=R^{n}$. We consider convolution of functions with a kernel of the form

$$
K_{\alpha}(x)=\frac{K_{n, \alpha}}{|x|^{n-\alpha}}, \quad 0<\alpha<n,
$$

where $|x|=\left(x_{1}^{2}+\cdots+x_{n}^{2}\right)^{1 / 2}, K_{n, \alpha}$ is a constant depending only on $n$ and $\alpha$. For the given choice of $\alpha$ choose any $P=\left(p_{1}, \ldots, p_{n}\right), 1<p_{i}<\infty, 1<i<n$, for which $\alpha=\sum \alpha_{i}, \alpha_{i}=1 / p_{i}^{\prime}, 1 / p_{i}^{\prime}+1 / p_{i}=1$. From the inequality $\left|x_{i}\right|<$ $\left(x_{1}^{2}+\cdots+x_{n}^{2}\right)^{1 / 2}, 1 \leqslant i \leqslant n$, it is not difficult to show that

$$
\frac{1}{|x|^{n-\alpha}}=\frac{1}{|x|^{\Sigma 1 / p_{i}}} \leqslant \frac{1}{\left|x_{1}\right|^{1 / p_{1}} \cdots\left|x_{n}\right|^{1 / p_{n}}} .
$$

This implies the kernel $K_{\alpha}(x)$ is in $L(P, \infty ; *)$ for $\alpha=n-\Sigma 1 / p_{i}$. We remark that in the $L(p, q)$ version [16], the kernel is shown to be in $L(p, \infty)$, where $p=$ $n /(n-\alpha)$. This is analogous to our special case $p_{1}=\cdots=p_{n}=n /(n-\alpha)$.

For a function $f(x)$ the ordinary fractional integral $f_{\alpha}$ of order $\alpha$ for the function $f$ is the ordinary convolution of $f$ with a kernel of the form $K_{\alpha}(x)$ (i.e., $f_{\alpha}=$ $\left.f * K_{\alpha}\right)$. Using the Convolution Theorem 5.3 and following the statements given above, the $L(P, Q ; *)$ analogue of the fractional integration theorem take the following form.

THEOREM 5.4. If $f \in L(P, Q ; *)$ on $R^{n}$ and $f_{\alpha}$ is its ordinary fractional integral of order $\alpha$ where $0<A<1 / P, A=\left(\alpha_{1}, \ldots, \alpha_{n}\right), \sum \alpha_{i}=\alpha$, then $f_{\alpha} \in L(R, Q ; *)$ where

I. $1 / R=1 / P-A$

II. $1 / R=1 / P-\alpha / n$, if $R=(r, \ldots, r), P=(p, \ldots, p)$.

5.4. Appendex. For the proof of Theorem 5.1 we use the ideas given in Hunt [11]. The key to the multivariate version is the use of rearrangeable simple functions to establish the lemma below. The lemma itself is a generalization of the first part of Hunt's proof. Once proof of the lemma is accomplished, the remainder of the proof proceeds as in [11] with only some minor modifications.

LEMMA 5.5. Suppose that $f(x), x=\left(x_{1}, x_{2}\right)$, is a rearrangeable simple function; then $f$ can be written in the form

$$
f(x)=e^{i \arg f(x)}\left(G_{0}(x)\right)^{1-\theta}\left(G_{1}(X)\right)^{\theta},
$$

where $G_{j}, j=0,1$, is a nonnegative simple function such that $\left\|G_{j}^{*}\right\|_{P_{j} Q_{j}}^{*} \leqslant$ $C\left(\left\|f^{*}\right\|_{P Q}^{*}\right)^{q_{2} / q_{2 j}, j=0,1 .}$

Proof. Setting our notation: $P=\left(p_{1}, p_{2}\right), P^{\prime}=\left(p_{1}^{\prime}, p_{2}^{\prime}\right), Q=\left(q_{1}, q_{2}\right), Q^{\prime}=$ $\left(q_{1}^{\prime}, q_{2}^{\prime}\right), P_{j}=\left(p_{1 j}, p_{2 j}\right), P_{j}^{\prime}=\left(p_{1 j}^{\prime}, p_{2 j}^{\prime}\right), Q_{j}=\left(q_{1 j}, q_{2 j}\right), Q_{j}^{\prime}=\left(q_{1 j}^{\prime}, q_{2 j}^{\prime}\right) ; j=0,1$. Let $0<r<\{R\}$, where $R$ is one of the indices given above. Let $t=\left(t_{1}, t_{2}\right)$ and put

$$
\begin{gathered}
\phi_{i j}\left(t_{i}\right)=t_{i}^{\left(1 / q_{i j}\right)\left(q_{i} / p_{i}-q_{i j} / p_{i j}\right)}, \\
F_{1 j}=f_{r}^{* *}(t)^{q_{1} / q_{1 j}}, \quad F_{2 j}=\left\|f_{r}^{* *}\left(\cdot, t_{2}\right)\right\|_{p_{1} q_{1}}^{* s_{1}},
\end{gathered}
$$

where $s_{j}=q_{2} / q_{2 j}-q_{1} / q_{1 j} ; i=0,1 ; j=0,1$. 
Let $h_{j}=\left(F_{1 j}\right)\left(F_{2 j}\right)\left(\phi_{1 j}\left(t_{1}\right)\right)\left(\phi_{2 j}\left(t_{2}\right)\right) ; j=0$, 1. We have $f_{r}^{* *}(t)=\left(h_{0}(t)\right)^{1-\theta}\left(h_{1}(t)\right)^{\theta}$. A direct computation and (4.2) implies

$$
\|h\|_{P_{j} Q_{j}}^{*} \leqslant C\left(\left\|f^{*}\right\|_{P Q}^{*}\right)^{q_{2} / q_{2 j}}, \quad j=0,1 \text {. }
$$

If $S k(t)=\left[\int_{t_{2}}^{\infty} \int_{t_{1}}^{\infty} k^{r}\left(t_{1}, t_{2}\right) d t_{1} d t_{2}\right]^{1 / r}$, then by the device of interchanging orders of integration it is not difficult to show that $f^{*}(t) \leqslant S f_{r}^{* *}(t)$. By Hölder's inequality $f^{*}(t) \leqslant\left(S h_{0}(t)\right)^{1-\theta}\left(S h_{1}(t)\right)^{\theta}$. After use of Minkowski's integral unequality to set up the integrals in proper order the Hardy inequalities [11] imply $\left\|S h_{j}\right\|_{P_{j} Q_{j}}^{*}<C\left\|h_{j}\right\|_{P_{j} Q_{j}}^{*}$ $\leqslant C\left(\left\|f^{*}\right\|_{P Q}^{*}\right)^{q_{2} / q_{2 j},} j=0$, 1 , where $C$ may be different in each inequality. The symbol $C$ will be used generically to represent constants which depend only on the indices.

The functions $G_{j}, j=0,1$, are obtained by choosing values smaller than $S h_{j}$, $j=0,1$. This is accomplished as follows. By Theorem 2.6

$$
f^{*}(t)=\sum_{n, m=1}^{N, M} k(n, m) \chi_{B^{*}(n, m)}(t),
$$

where $B^{*}(n, m)=[\alpha(n-1), \alpha n) \times[\beta(m-1), \beta m)$. For a given $t, f^{*}(t)=k(n, m)$ uniquely for some $n, m$. Since $S h_{j}(t)$ is continuous, $k(n, m)<$ $\left(S h_{0}(\alpha n, \beta m)\right)^{1-\theta}\left(S h_{1}(\alpha n, \beta m)\right)^{\theta}$. Define

$$
\begin{aligned}
G_{0}(X) & =\sum S h_{0}(\alpha n, \beta m) \chi_{B(n, m)}(x), \\
G_{1}(x) & =\sum \frac{(K(n, m))^{1 / \theta}}{\left(S h_{0}(n, m)\right)^{(1-\theta) / \theta}} \chi_{B(n, m)}(x) .
\end{aligned}
$$

This implies $f=\left(G_{0}\right)^{1-\theta}\left(G_{1}\right)^{\theta}$. Note that each term in $G_{1}$ is majorized by $S h_{1}(\alpha n, \beta m)$. Put $\bar{G}_{1}(x)=\sum S h_{1}(\alpha n, \beta m) \chi_{B(n, m)}(x)$; then $G_{1}(x)<\bar{G}_{1}(x)$ and $G_{1}^{*}(t)$ $\leqslant \bar{G}_{1}^{*}(t)$. By construction, the functions $G_{0}(X)$ and $\bar{G}_{1}(x)$ are rearrangeable simple functions and as written are in rearranged form. Term by term the underlying sets, associated coefficients and how they are ordered matches up with that for the function $f(x)$. This implies

$$
G_{0}^{*}(t)=\sum S h_{0}(\alpha n, \beta m) \chi_{B^{*}(n, m)}(t)
$$

and similarly for $\bar{G}_{1}^{*}(t)$. Moreover, by continuity of the defining integrals we have $G_{0}^{*}(t) \leqslant S h_{0}(t)$ and $\bar{G}_{1}^{*}(t) \leqslant S h_{1}(t)$. This proves the lemma.

Proof of Theorem 5.1. We prove the case $n=m=2$. Let $G_{j}, j=0,1$, be as in Lemma 5.5. Put

$$
F(x, Z)=e^{i \arg f(x)}\left[G_{0}(x)\right]^{1-Z}\left[G_{1}(x)\right]^{Z},
$$

$Z$ complex, $0 \leqslant R(Z) \leqslant 1, x=\left(x_{1}, x_{2}\right)$. This expression compares to that given in Hunt [11, p. 267]. By working primarily with averaging operators of the type $\bar{f}_{r}$, the reader should have little difficulty in making use of the subsequent steps given there in order to obtain the result $\left\|(T f)^{*}\right\|_{P^{\prime} Q^{\prime}}^{*} \leqslant C C_{0}^{1-\theta} C_{1}^{\theta}\left\|f^{*}\right\|_{P Q^{\prime}}^{*}$, where $f$ is any rearrangeable simple function. We omit the details.

For any function $f \in L(P ; Q ; *)$, find a sequence of rearrangeable simple functions $f_{k}$ converging to $f$ in metric (see \$2.2) and do the following. Let $A_{N} \subset \Gamma$ 
be of the form $A_{N}=A_{1 N} \times A_{2 N}$, where $\left|A_{i N}\right|<\infty, i=1,2$, and $A_{N} \uparrow \Gamma$. The result above implies that $T$ maps rearrangeable simple functions in $L(P, Q ; *)$ into measurable functions in $L\left(P^{\prime}, Q^{\prime} ; *, A_{N}\right)$, which is a complete space. If necessary by passing to a subsequence we obtain $\left\|f_{k}^{*}\right\|_{P Q}^{*} \rightarrow\left\|f^{*}\right\|_{P Q}^{*}$ and $\left|T f_{k}\right| \rightarrow|T f|$ pointwise a.e. $y$ in $A_{N}$. By Fatou's lemma.

$$
\left\|(T f)_{r}^{* *}\right\|_{P^{\prime} Q^{\prime}}^{*} \leqslant \lim \inf \left\|\left(T f_{k}\right)_{r}^{* *}\right\|_{P^{\prime} Q^{\prime}}^{*}
$$

This implies $\left\|(T f)^{*}\right\|_{P^{\prime} Q^{\prime}}^{*} \leqslant C C_{0}^{1-\theta} C_{1}^{\theta}\left\|f^{*}\right\|_{P Q^{\prime}}^{*}$. After letting $A_{N} \uparrow \Gamma$ the proof is complete for the case $n=m=2$.

In the steps above it was not explicitly shown that convergence in metric gives the existence of a subsequence which converges pointwise almost everywhere. It follows from (4.4) that the function space in question is contained in $L^{r}\left(A_{n}\right)$, $r<P^{\prime}$. This implies almost everywhere pointwise convergence of a subsequence.

For the case of dimension $n>2, m \geqslant 1$, the proof is obtained by extending the definitions for the simple functions along with Theorem 2.6 and adapting the proof above to the $n$-dimensional context, $n>2$. The process, though tedious, is straightforward. The details are left to the discretion of the reader.

\section{REFERENCES}

1. A. Benedek and R. Panzone, The spaces $L^{P}$ with mixed norm, Duke Math. J. 26 (1961), 301-324.

2. C. Bennett and K. Rudnick, On Lorentz-Zygmund spaces, Dissertationes Math. (to appear).

3. A. P. Blozinski, On a convolution theorem for $L(p, q)$ spaces, Trans. Amer. Math. Soc. 164 (1972), 255-265.

4. ___ Averaging operators and Lipschitz spaces, Indiana Univ. Math. J. 26 (1977), 939-950.

5. P. L. Butzer and H. Berens, Semigroups of operators and approximation, Springer-Verlag, Berlin and New York, 1967.

6. M. Cwikel, On $\left(L^{P_{\mathrm{o}}}\left(A_{0}\right), L^{P_{1}}\left(A_{1}\right)\right)_{\theta, q}$, Proc. Amer. Math. Soc. 44 (1974), 286-292.

7. C. Ballester de Pereyra, Sobre la continuidad debil y magra en $L^{p}\left(L^{q}\right)$ y su aplicacion a operadores potencials, Tese de Doutorado, Universidad de Buenos Aires, 1963.

8. D. L. Fernandez, Lorentz spaces, with mixed norms, J. Funct. Anal. 25 (1977), 128-146.

9. P. R. Halmos, Measure theory, Van Nostrand, New York, 1950.

10. G. H. Hardy, J. E. Littlewood and G. Pólya, Inequalities, University Press, Cambridge, 1967.

11. R. A. Hunt, On $L(p, q)$ spaces, Enseign. Math. 12 (1966), 249-276.

12. W. A. J. Luxemburg, Banach functions spaces, Thesis (Defft), Assen, 1955.

13. Math. 10 (1967), 83-144.

14. M. Milman, Embeddings of $L(p, q)$ spaces and Orlicz spaces with mixed norms, Notas de Math., No. 13, Univ. de Los Andes, 1977.

15. __ Some new function spaces and their tensor products, Notas de Math., No. 20, Univ. de Los Andes, 1978.

16. R. O'Neil, Convolution operators and $L(p, q)$ spaces, Duke Math. J. 30 (1963), 129-142.

17. Integral transforms and tensor products on Orlicz spaces and $L(p, q)$ spaces, J. Analyse Math. 21 (1968), 1-276.

18. R. S. Schatten, $A$ theory of Cross spaces, Ann. Math. Studies, no. 26, Princeton, N. J., 1950.

19. R. Sharpley, Spaces $\Lambda_{\propto}(X)$ and interpolation, J. Funct. Anal. 11 (1972), 479-513.

20. E. M. Stein, Singular integrals and differentiability properties of functions, Princeton Univ. Press, Princeton, N. J., 1970.

21. A. Torchinsky, Interpolation of operations and Orlicz classes, Studia Math. 59 (1976), 177-207.

Texas A\&M University at Galveston, P. O. Box 1675, Galveston, Texas 77553 